\title{
Annealing Effect on Martensitic Transformation and Magneto-Structural Properties of Ni-Mn-In Melt Spun Ribbons
}

\author{
T. Sánchez ${ }^{1}$, J.L. Sánchez Llamazares ${ }^{1, a}$, B. Hernando ${ }^{1, b}$, J.D. Santos ${ }^{1}$, M.L. \\ Sánchez ${ }^{1}$, M.J. Perez ${ }^{1}$, J.J. Suñol ${ }^{2}$, R. Sato Turtelli ${ }^{3}$, and R. Grössinger ${ }^{3}$ \\ ${ }^{1}$ Departamento de Física, Universidad de Oviedo, Calvo Sotelo s/n, 33007 Oviedo, Spain \\ ${ }^{2}$ Universidad de Girona, Campus de Montilivi, edifici PII, Lluís Santaló s/n. 17003 Girona, Spain \\ ${ }^{3}$ Institut fur Festkörperphysik, TU Wien, Wiedner Hauptstr. 8-10, 1040 Vienna, Austria \\ asanchez@nanomagnetics.org, bgrande@uniovi.es
}

Keywords: Heusler alloys, melt spinning, martensitic transformation.

\begin{abstract}
We report the effect of a short-time vacuum annealing (1073 K during 10 minutes) on structural phase transition temperatures and magneto-structural properties of as-quenched ribbons of the Heusler alloy $\mathrm{Ni}_{50.6} \mathrm{Mn}_{34.5} \mathrm{In}_{14.9}$. This alloy crystallizes in a single phase cubic B2-type austenite with a Curie point of $T_{C}{ }^{A}=284 \mathrm{~K}$ that with the lowering in temperature transforms into a martensite with $T_{C}{ }^{M} \approx 185 \mathrm{~K}$. The direct and reverse martensitic phase transition temperatures were $M_{S}=257 \mathrm{~K}$, $M_{f}=221 \mathrm{~K}, A_{S}=239 \mathrm{~K}$, and $A_{f}=266 \mathrm{~K}$. After annealing austenite shows the highly ordered L2 $1^{-}$ type structure while the average chemical composition as well as the structural and magnetic transition temperatures were shifted to $\mathrm{Ni}_{50.2} \mathrm{Mn}_{34.3} \mathrm{In}_{15.5}$ and $M_{S}=253 \mathrm{~K}, M_{f}=238 \mathrm{~K}, A_{S}=257 \mathrm{~K}$, $A_{f}=265 \mathrm{~K}, \Delta T=13 \mathrm{~K}, T_{C}{ }^{A}=299 \mathrm{~K}$ and $T_{C}{ }^{M} \approx 207 \mathrm{~K}$. In the annealed samples the magnetization changes associated to the magnetic and structural transitions are more abrupt and magnetization isotherms in both the austenitic and martensitic existence region show higher initial magnetic susceptibility and faster approach to saturation. Field-cooled hysteresis loops at $10 \mathrm{~K}$ were shifted along the negative $H$-axis for both samples, but a significant anomaly was evident on the left side of the hysteresis loop for as-quenched ribbons.
\end{abstract}

\section{Introduction}

Recently, it has been reported in literature that ferromagnetic shape memory alloys in the Heusler $\mathrm{Ni}_{50} \mathrm{Mn}_{50-\mathrm{y}} \mathrm{X}_{\mathrm{y}}(\mathrm{X}=\mathrm{Sn}, \mathrm{In})$ systems can be produced as single-phase materials by rapid solidification using melt spinning technique [1,2]. The ribbon flakes obtained usually crystallize in a cubic L $2_{1^{-}}$ type austenitic phase. Upon cooling austenite undergoes a martensitic transformation into an orthorhombic or monoclinic martensite for Sn- and In-based alloys, respectively. Xuan et al. studied the effect of a 10 minutes thermal treatment at $1123 \mathrm{~K}\left(850^{\circ} \mathrm{C}\right)$ and $1173 \mathrm{~K}\left(900^{\circ} \mathrm{C}\right)$ on the martensitic phase transformation temperature, magnetocaloric properties and magnetoresistance of $\mathrm{Ni}_{44.1} \mathrm{Mn}_{44.2} \mathrm{Sn}_{11.7}$ ribbons [3,4]. They found an increase of both, the temperature at which martensite starts its transformation into austenite $A_{S}$ and the Curie point of austenite $T_{C}{ }^{A}$. The shift of $A_{S}$ after the $1173 \mathrm{~K}\left(900^{\circ} \mathrm{C}\right)$ annealing was considerable (around $\left.40 \mathrm{~K}\right)$. The results were ascribed to the stress and structural relaxation of samples upon annealing. Here we present a preliminary study of annealing influence $\left(1073 \mathrm{~K}\left(800^{\circ} \mathrm{C}\right)\right.$ for 10 minutes $)$ on the structural and magnetic properties of as-quenched $\mathrm{Ni}-\mathrm{Mn}$-In ribbons produced by melt spinning technique.

\section{Experimental}

The master alloys for ribbon production using melt spinning technique were as-cast ingots previously obtained by argon arc melting from highly pure elements $(99.98 \%$ pure $\mathrm{Ni}, 99.98 \% \%$ pure Mn, and $99.999 \%$ pure In). The samples were melted several times to ensure their chemical composition homogeneity. A detailed description of the procedure followed to produce melt spun ribbons can be found elsewhere [2]. These Heusler alloys show a fast crystallization kinetic and the 
ribbons produced (even at high speeds of the rotating copper wheel) show a microstructure composed by micronic columnar grains with their longer axis aligned perpendicular to ribbon plane [2]. Hence, ribbons are very brittle and during solidification process become continuously fragmented. The ribbon flakes obtained were of typically 4-7 $\mathrm{mm}$ in length and 1.5-2.0 $\mathrm{mm}$ in width. Samples were annealed in high vacuum for 10 minutes at $1073 \mathrm{~K}\left(800^{\circ} \mathrm{C}\right)$ in closed quartz ampoules. Annealing was followed by water quenching.

Elemental chemical composition was studied by means of a scanning electron microscopy equipped with an X-ray energy dispersive spectroscopy (EDS) system. Ribbon thickness varied between $9 \mu \mathrm{m}$ and $12 \mu \mathrm{m}$. In order to determine the average elemental composition an appreciable number of ribbon flakes were examined. After more than 70 analyses on the ribbon cross section we obtained for the as-quenched and annealed ribbons the average chemical composition $\mathrm{Ni}_{50.6} \mathrm{Mn}_{34.5} \mathrm{In}_{14.9}$ and $\mathrm{Ni}_{50.2} \mathrm{Mn}_{34.3} \mathrm{In}_{15.5}$, respectively. X-ray diffraction (XRD) analyses were performed at room temperature using $\mathrm{Cu}-\mathrm{K} \alpha$ radiation $(\lambda=1.5418 \AA$ ) in a Phillips X'pert PRO (XP2) powder diffractometer. The X-ray diffraction patterns were measured by exposing the free surface of ribbons to the $\mathrm{X}$-ray incident radiation.

Magnetic measurements were done using a physical properties measuring system platform with the vibrating sample magnetometer module (Quantum Design). Zero-field cooling (ZFC), field cooling (FC), and field heating (FH) magnetization curves were recorded heating from $4 \mathrm{~K}$ to $330 \mathrm{~K}$ at $50 \mathrm{Oe}$. A heating or cooling rate of $1.5 \mathrm{~K} / \mathrm{min}$ was employed. Curie point of the magnetic phases formed was inferred from the minimum in the $d M / d T$ versus $T$ curve. The field was applied along the ribbon axis (i.e. rolling direction) to minimize demagnetizing field effect.

\section{Results and discussion}

Figure 1 shows the characteristic ZFC, FC and FH $M(T)$ curves of the as-quenched and annealed alloy ribbons. The inserts show the normalized $d M / d T(T)$ curves corresponding either to the reverse and direct martensitic phase transition (upper graph) and the ferromagnetic-paramagnetic transition of austenite (lower graph). $d M / d T$ has been normalized to its maximal (or minimal) value and the temperature axis to the temperature value at which $d M / d T$ reaches this maximum (or minimum). The figure shows the general behavior of the $M(T)$ curves for both samples is rather similar. The magnetic and structural transitions caused changes in the magnetization of the alloy. Hence,

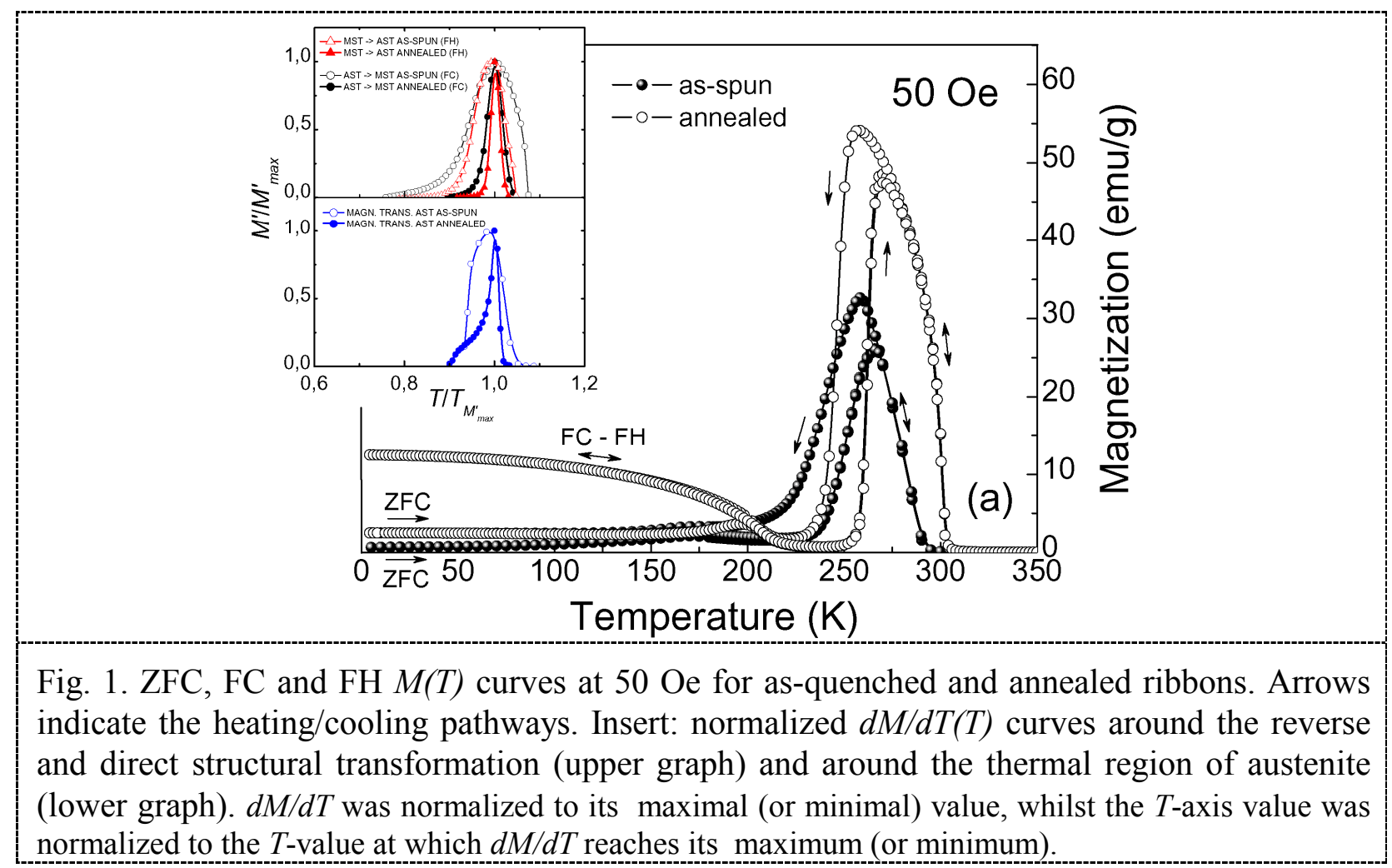


following the ZFC $M(T)$ curve we can describe them. The first event we note is a smooth and broad transition around $200 \mathrm{~K}$ that can be attributed to the ferromagnetic transition of the martensite, a few tens of Kelvin degrees over the abrupt raise in magnetization reveals the occurrence of the firstorder reverse martensitic transformation (the transformation shows a thermal hysteresis $\Delta T$ as the corresponding FC curve indicates). At last the ferromagnetic-paramagnetic transition of austenite leads to a second drop in magnetization. From a comparison of the transitions occurred in both curves it can be observed that the changes in magnetization associated to any of them are more abrupt in the annealed sample. The latter is further illustrated by the narrower $d M / d T(T)$ curves obtained. Moreover, it must be noted that both $A_{S}$ and the magnetic transition temperatures increase for the annealed sample. The structural and magnetic phase transition temperatures are given in Table 1 . In these alloys the $\mathrm{Mn}$ atoms that exceed the stoichiometric composition $\mathrm{Ni}_{2} \mathrm{MnIn}$ occupy a fraction of the In sites in the L2 ${ }_{1}$-type crystal structure of austenite giving rise to some form of antiferromagnetic (AFM) coupling [5,6]. Below the martensitic transformation temperature the change in the interatomic distances among neighbouring $\mathrm{Mn}$ atoms in In sites and those occupying regular Mn sites lead to the strengthening of this AFM coupling. It has been stated in literature that the coexistence of AFM and ferromagnetic (FM) interactions within martensite is macroscopically evidenced by its lower saturation magnetization with respect to austenite, the observed splitting between the ZFC and FC pathways of the temperature dependence of the low-field magnetization [7], and the exchange bias phenomenon reported at low temperatures for these alloys [8,9]. The more abrupt change in magnetization in the thermal region where magnetic transitions occur as well as the observed increase in $T_{C}{ }^{M}$ and $T_{C}{ }^{A}$ must result from the strengthening of $\mathrm{FM}$ coupling. Therefore, our results put in evidence how the atomic ordering and the atomic rearrangement caused by annealing affect such coexisting FM and AFM correlations and their mutual balance.

Table 1. Characteristic phase transition temperatures and thermal hysteresis (determined as $A_{f}-M_{S}$ ) for as-quenched and annealed ribbons determined from the $M(T)$ curve.

\begin{tabular}{llllllll}
\hline Sample & $A_{S}[\mathrm{~K}]$ & $A_{f}[\mathrm{~K}]$ & $M_{S}[\mathrm{~K}]$ & $M_{f}[\mathrm{~K}]$ & $\Delta T[\mathrm{~K}]$ & $T_{C}{ }^{M}[\mathrm{~K}]$ & $T_{C}{ }^{A}[\mathrm{~K}]$ \\
\hline as-quenched & 239 & 266 & 257 & 221 & 9 & 185 & 284 \\
annealed & 257 & 265 & 253 & 238 & 13 & 207 & 299 \\
\hline
\end{tabular}

Figures 2(a) and (b) show the room temperature XRD patterns of the as-quenched and annealed ribbon, respectively. They were satisfactorily indexed on the basis of the cubic B2 [a=0.2994(2)] $\mathrm{nm}$ and $\mathrm{L} 2{ }_{1}$-type $[a=0.5987(4)]$ structures, respectively. Traces of secondary or spurious phases were not detected, so in both samples a single-phase austenite is formed. The result for the asquenched sample differs from the previously reported for alloy of similar composition where L $2_{1}$ structure crystallized from the melt [1]. Thus, the high quenching rates leads to the formation of ordered B2 structure meanwhile a short annealing at $1073 \mathrm{~K}\left(800^{\circ} \mathrm{C}\right)$ favors the formation of stable highly ordered cubic L2 $2_{1}$ structure (which is confirmed by observation of the 111 and 311 superstructure peaks in the diffraction pattern). Thus, the austenite of as-quenched sample has lower chemical order. The rapid change observed in the $M(T)$ curves for the magnetic transition of austenite in the annealed ribbons is in agreement with a high chemical order in the occupancy of Mn and In atoms in the crystal structure having a direct influence on the exchange interactions distribution. Only minor differences between peak intensities were found from the comparison of the diffraction patterns of both sides of the ribbon. 


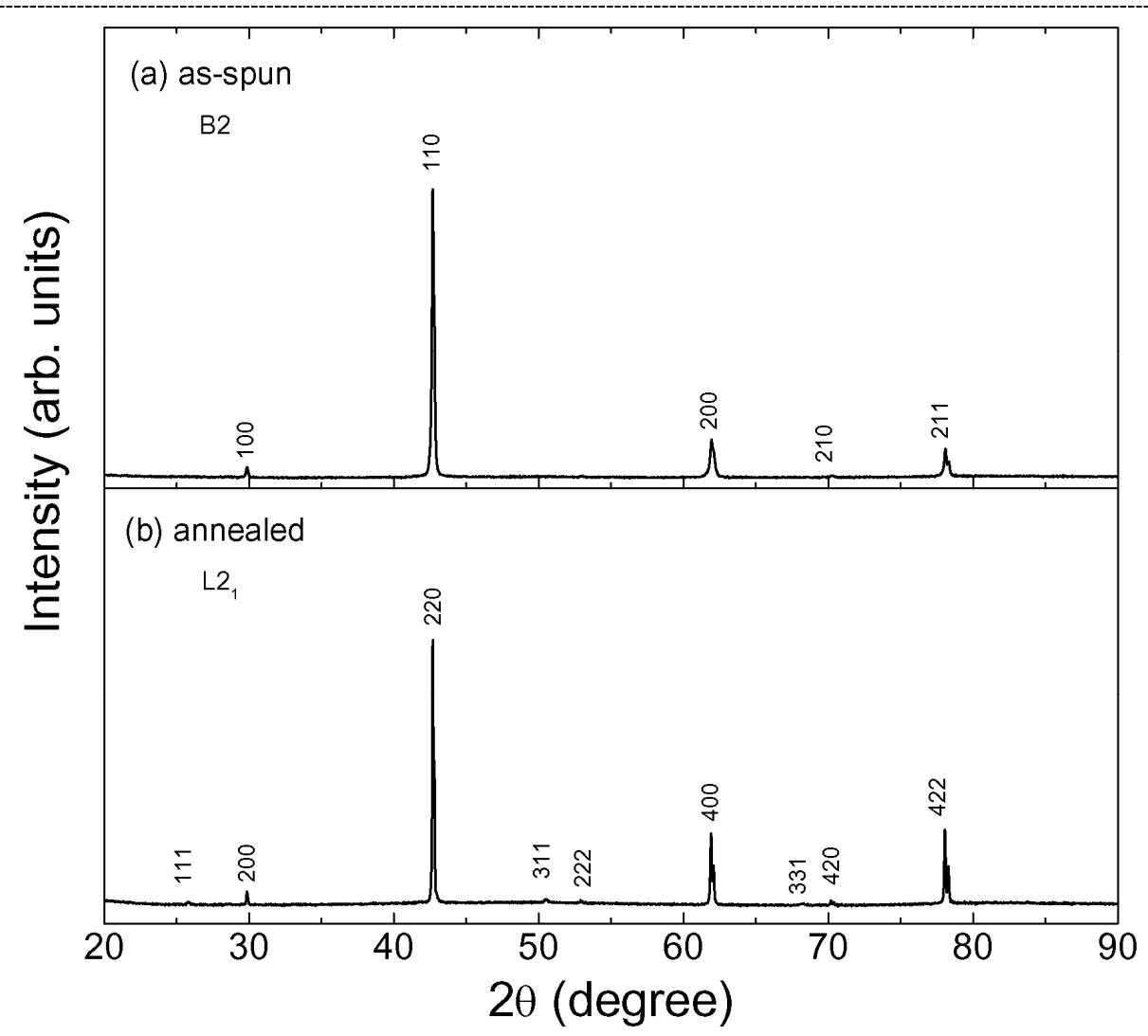

Fig. 2. Room temperature XRD patterns for as-quenched (a) and annealed (b) ribbons.

In figures 3(a) and (b) we compare the magnetization curves of martensite and austenite phases at a constant value of the ratio between the measuring temperature and the magnetic transition of both phases (i.e. $T / T_{C}{ }^{M}$ and $T / T_{C}{ }^{A}$, respectively. The temperature at which each curve was measured is given in the figure). In addition, the $M$-axis has been normalized to the $M$ value reached at $20 \mathrm{kOe}$. As the figure points out, the $M(H)$ curves for martensite and austenite in the annealed sample are characterized by a high initial susceptibility and fast approach to saturation suggesting that in the low-field region domain walls easily displace. Therefore, magnetization process is governed by domain wall displacements. Nevertheless, a quite different behavior is shown by the martensite in the as-quenched ribbons. After an initial increase in the magnetization up to a field around $1.5 \mathrm{kOe}$, almost linear approaching to saturation level is obtained. This behavior could be ascribed to the coexistence of two different orientations of the easy anisotropy axis in martensite with respect to the length of the ribbon. Here we must consider that the low temperature martensitic phase formed is derived from an ordered B2 cubic parent phase (having chemical and/or topological short range order inhomogeneities). As a consequence a resulting non-collinearity of the easy anisotropy axes of AFM and FM components within the martensite is developed. Also note that at the maximum field applied, $H_{\max }=20 \mathrm{kOe}$, the sample is still far from saturation. Upon annealing an important increase in the saturation magnetization value at $H_{\max }=20 \mathrm{kOe}$ of martensite from $27.8 \mathrm{emu} / \mathrm{g}$ to $34.7 \mathrm{emu} / \mathrm{g}$ was observed.

Figure 4(a) shows the low-field region of FC hysteresis loops for both samples at $10 \mathrm{~K}$ (-1500 Oe $\leq H \leq+1500$ Oe), while the whole loops are shown in the insert. First it must be noted that both hysteresis loops are shifted along the negative $H$-axis denoting the occurrence of exchange bias effect [8-10], but that of the annealed one shows regular magnetization paths whereas the demagnetizing curve in the second-to-third quadrant for the as-quenched sample shows a welldefined anomaly. The anomaly reveals the existence of an asymmetric magnetization reversal processes. The intrinsic character of this phenomenon was verified by recording twice the loop. The figure 5 shows how the subsequent reversals of magnetization do not affect the hysteresis loops (i.e. 
a first and second loops consecutively recorded overlap). A similar result was obtained measuring the loop under a $50 \mathrm{kOe}$ magnetic field. The origin of this behavior is under study.

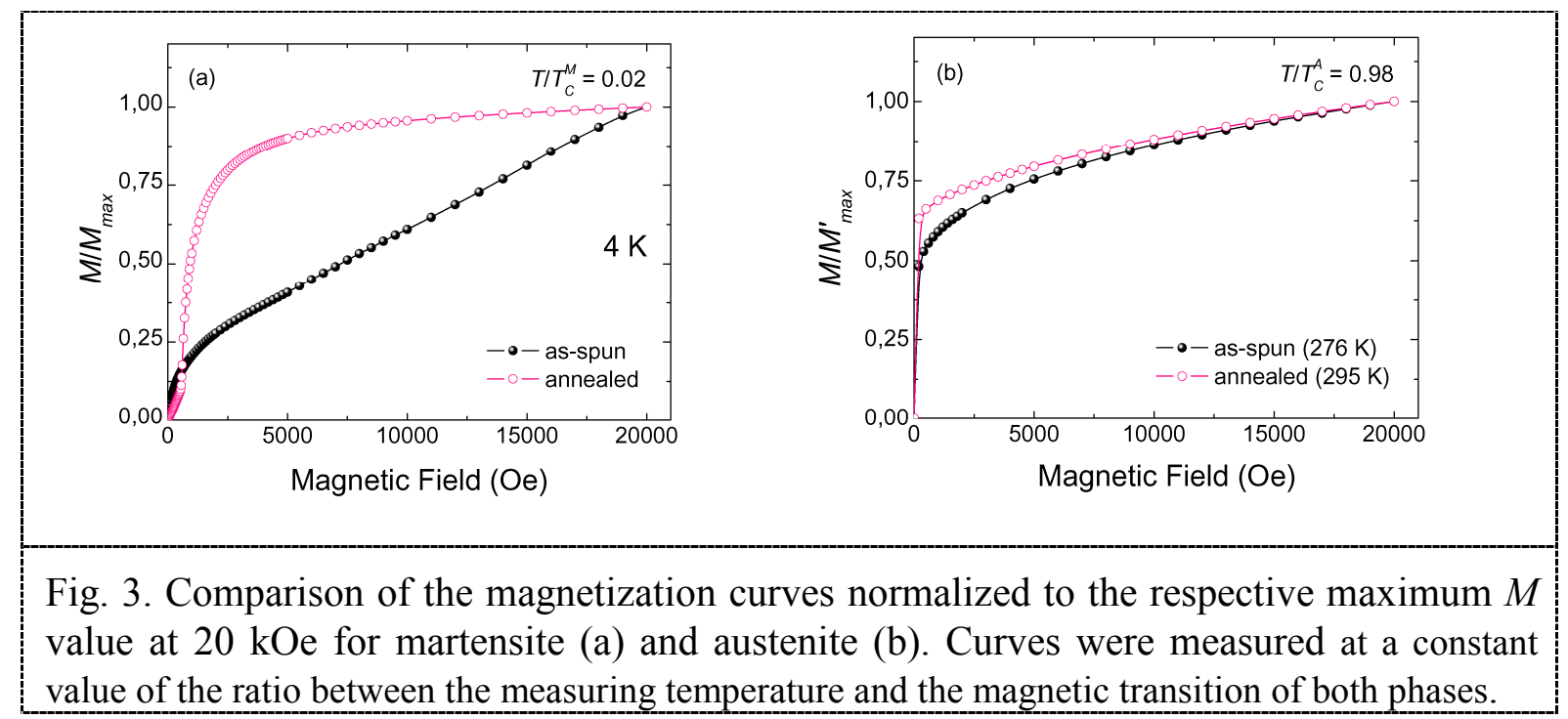

Figure 4(b) compares the ZFC hysteresis loops of both samples at $10 \mathrm{~K}$. The loops up to $H_{\max }=$ $20 \mathrm{kOe}$ are shown in the insert. Note that the anomaly of the hysteresis loop for the as-quenched samples is stressed and now it appears in both, the left and right side of the loop. The initial $M(H)$ curve shows the characteristic change in the slope followed by an almost linear dependence previously described for the sample at $4 \mathrm{~K}$. As the insert shows the field is no enough to saturate the sample. However, the ZFC loops of both samples can be considered double-shifted rather than asymmetric. Such double-shifted hysteresis loops have been observed in bulk Ni-Mn-In [10] and Ni-Mn-Sn [11] alloys and are observed in certain materials with coexisting AFM and FM layers (see e.g. Ref. [11] and reference therein).

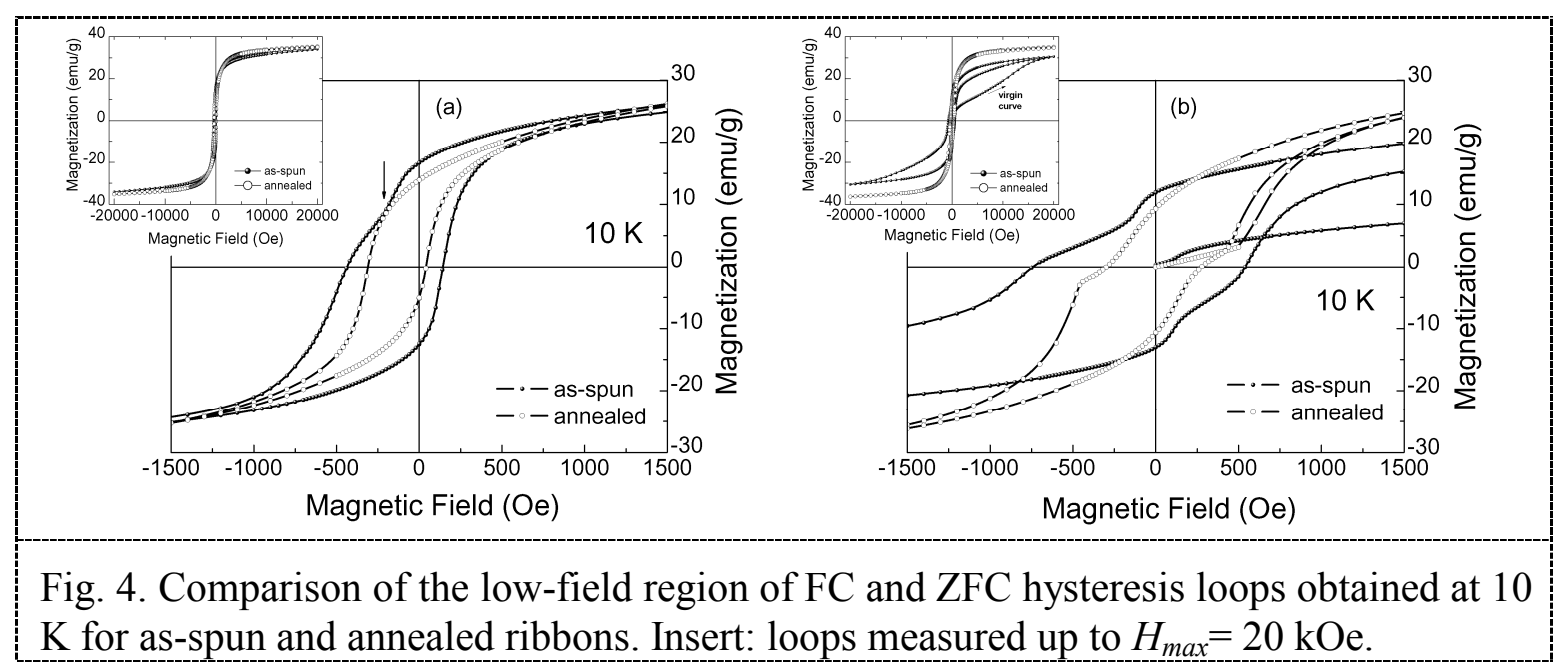




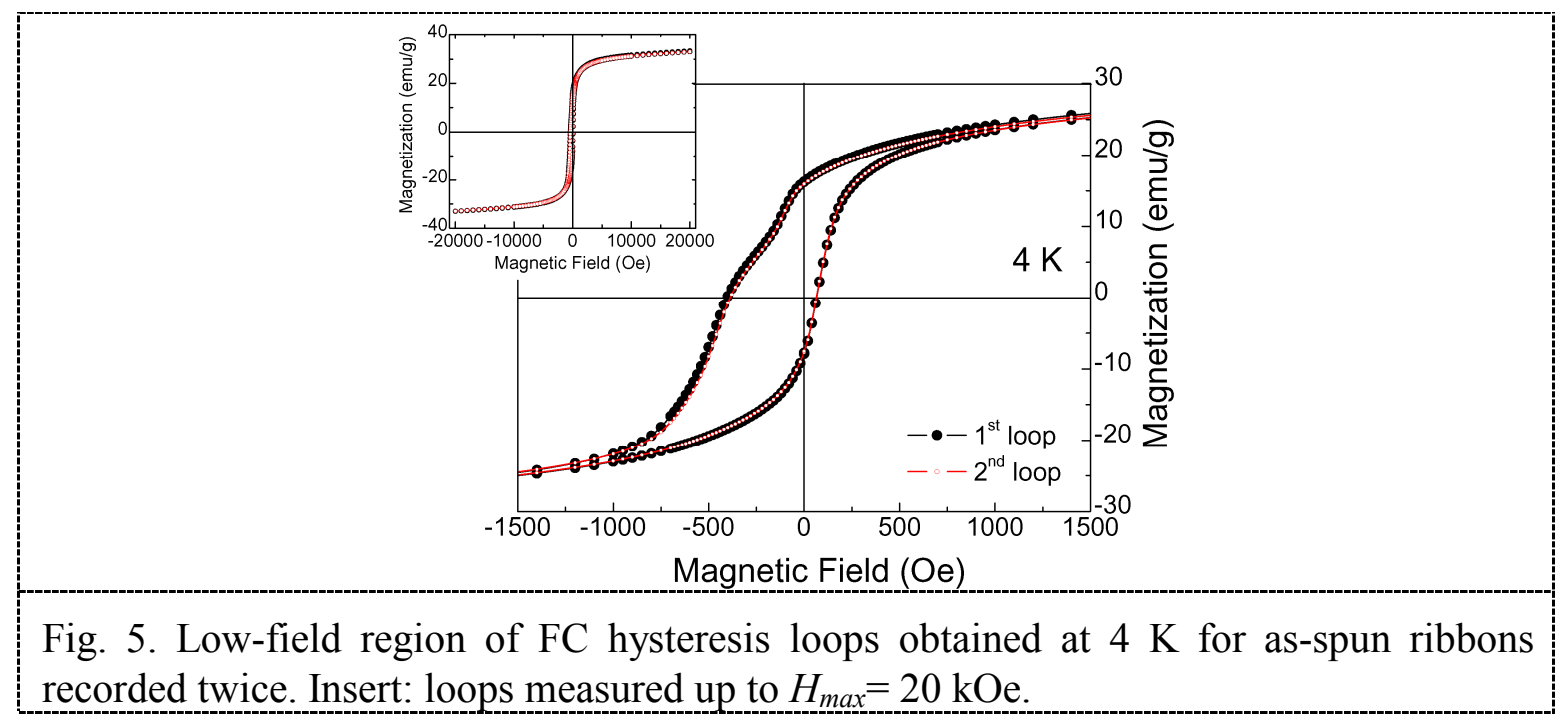

\section{Conclusions}

We present a preliminary study on the effect of vacuum annealing during 10 minutes at $1073 \mathrm{~K}$ on the crystal structure of austenite, structural and magnetic phase transition temperatures and magnetic properties of as-quenched ribbons of the ferromagnetic shape memory alloy $\mathrm{Ni}_{50.6} \mathrm{Mn}_{34.5} \mathrm{In}_{14.9}$. In as-quenched alloys austenite crystallizes in the ordered B2-type crystal structure whereas the short annealing performed favors the formation of a highly ordered L2 $2_{1}$-type structure. Broader transitions associated to the structural and magnetic transitions were observed in as-quenched samples in the low-field $M(T)$ curve. The observed exchange bias behavior for martensite confirmed the coexistence of AFM and FM exchange interactions in martensite phase. The atomic and chemical rearrangements associated to the structural relaxation induced by thermal annealing reinforce FM coupling leading to the observed increase in the magnetic ordering temperature of austenite and martensite.

\section{Acknowledgements}

Present research was carried out under the supporting Spanish grant MAT2009-13108-C02-01-02 (MICINN). J.L. Sánchez Llamazares (COF07-013) and T. Sánchez (BP07-075) acknowledge FICYT. Authors thank to M. Vazquez (ICMM-CSIC) for sample preparation facilities.

\section{Literature References}

[1] J.L. Sánchez Llamazares, B. Hernando, C. García, J. González, Ll. Escoda, J.J. Suñol, J. Phys. D: Appl. Phys., 42 (2009) 045002.

[2] J. D. Santos, T. Sanchez, P. Alvarez, M. L. Sanchez, J. L. Sánchez Llamazares, B. Hernando, L1. Escoda, J. J. Suñol, and R. Varga, J. Appl. Phys. 103 (2008) 07 B326.

[3] H. C. Xuan, K. X. Xie, D. H. Wang, Z. D. Han, C. L. Zhang, B. X. Gu, Y. W. Du, Appl. Phys. Lett. 92 (2008) 242506.

[4] H. C. Xuan, Y. Deng, D. H. Wang, C. L. Zhang, Z. D. Han, Y. W. Du, J. Phys. D: Appl. Phys., $41(2008) 215002$.

[5] P. J. Brown, A. P. Gandy, K. Ishida, R. Kainuma, T. Kanomata, K.U. Neumann, K. Oikata, B. Ouladdiaf, K. R. A. Ziebeck J. Phys.: Condens. Matter 18, 2249 (2006).

[6] S. Aksoy, M. Acet, P. P. Deen, L. Mañosa, A. Planes, Phys. Rev. B 79, 212401 (2009). 
[7] T. Krenke, M. Acet, E.F. Wassermann, X. Moya X, L. Mañosa, A. Planes Phys. Rev. B 73, 174413 (2006).

[8] A. Kumar, M. Khan, B. R. Gautam, S. Stadler, I. Dubenko, and N. Ali, J. Magn. Magn. Mater. 321, 963 (2008).

[9] Chao Jing, Jiping Chen, Zhe Li, Yanfei Quiao, Baojuan Kang, Shixun Cao, and Jincang Zhang, J. Alloys Compd. 475, 1 (2009).

[10] B. M. Wang, Y. Liu, L. Wang, S.L. Huang, Y. Zhao, Y. Yang, and H. Zhang, J. Appl. Phys. 104, 043916 (2008).

[11] Khan Mahmud, Dubenko Igor, Stadler Shane, Ali Nausead, J. Appl. Phys. 102, 113914 (2007). 